\title{
Perspective of Making Self-training Habit from Psychological Consideration and Practice
}

\section{Makoto Takasugi ${ }^{1}$, Akito Moriyasu ${ }^{2}$, Hiroshi Bando ${ }^{3,4 *}$, Hiroya Hanabusa $^{2}$ and Mitsuru Murakami ${ }^{5}$}

${ }^{1}$ Home-visit Nursing Station Miki, Kagawa, Japan

${ }^{2}$ Akiboshi Bright Star Training Rehabilitation Center, Kagawa, Japan

${ }_{3}^{3} J a p a n$ Masters Athletics, Tokushima Division, Board, Tokushima, Japan

${ }^{4}$ Tokushima University/Medical Research, Tokushima, Japan

${ }^{5} J a p a n$ Masters Athletics, Kagawa Division, Vice-president, Kagawa, Japan

*Corresponding Author: Hiroshi Bando, Japan Masters Athletics, Tokushima

Division and Tokushima University/Medical Research, Tokushima, Japan.
Received: January 13, 2021

Published: Janaury 28, 2021

(C) All rights are reserved by Hiroshi Bando., et al.

\section{Abstract}

Authors and collaborators have continued clinical practice and research on rehabilitation and self-training, in which various problems were found.

Protocol: The author himself tried home self-training exercise of push-up for 2 months, which was successfully achieved.

Results: Positive changes were 94 to $96.5 \mathrm{~cm}$ in chest circumference, and 45 to 100 times in continuous push-up, respectively.

Discussion: From the viewpoint of sport psychology, close relationship among motivation, self-efficacy and performance has been observed. Self-efficacy can influence one's beliefs concerning accomplishing and continuing the task, activities and effort. This report will hopefully become the reference for future practice and research development.

Keywords: Self-training; Push-up; Motivation; Self-efficacy; Rehabilitation

\section{Introduction}

In various types of rehabilitation, to continue self-training has been necessary on a daily basis [1,2]. However, it is often found that the patients cannot continue enough self-training which was proposed by physiotherapists [3]. What kinds of factors bring this situation? In order to clarify the related causes, it is necessary to investigate the detail of the motivation and ingenuity to continue $[4,5]$.

The author and collaborators have been involved in medical practice, rehabilitation, sports medicine, masters' sports, and so on [6]. We have also continued several research from various points of view and reported on medical journals [7]. Among them, the au- thor has come to consider clinical problem for the continuation of self-training [8] In fact, some cases can continue with satisfactory results, and others may discontinue with unsatisfactory course [9]. The author has made a certain attempt using author himself as the target case, and report it with some consideration in this article.

\section{Protocol}

The subject was the author himself, and the method was pushup for strength training [10]. The reason is described in the following.

- The number of times and the degree of load can be quantified, and the bulge of the muscle can be observed. One of the personal goals for the subject is to feel confidence in the muscular body when going to sauna bath. 
- It can be practiced anytime and anywhere, and the number of times can be increased with a low load. Some music can be accompanied at the same time for feeling the fun.

- To leave the image of the body is possible on a regular basis. Thus, the obtained data can be compared visually and indicated the effect.

- As the actual method, push-ups are performed with the elbow angle bending by about $30^{\circ}$ from the starting position. One set was carried out 20 times, with 5 sets for a total of 100 times, every day [10]. The load amount and number of times exceeding the target can be increased, where it can be allowed freely, but it cannot be rested the next day with the necessary condition.
- The trial period is 8 weeks from August to October, 2020 [11]. The evaluations were made in (1) chest circumference, (2) number of consecutive push-ups, and (3) image comparison.

\section{Results}

The following data were obtained in the 0,4 , and 8 weeks of this project. The chest circumference was $94 \mathrm{~cm}, 94 \mathrm{~cm}, 96.5 \mathrm{~cm}$, and the number of continuous push-ups was $45,70,100$, respectively. Comparing the chest images, a macroscopic increase in the muscle mass was observed (Figure 1). This practice could be continued during 2 months, and the positive changes were observed functionally and objectively.

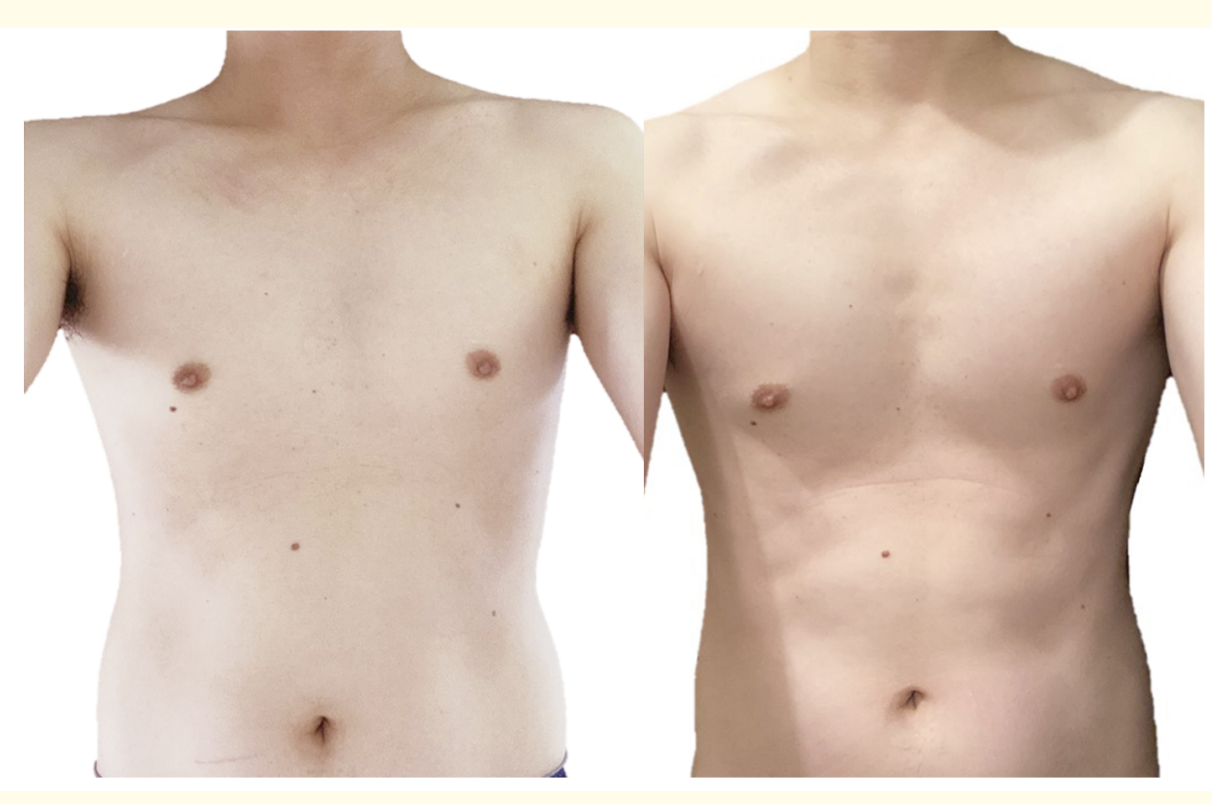

Figure 1: Changes in chest muscles before and after the home self-training on push up.

At the beginning, additional way to motivate the exercise with music was conducted [12]. However, it could be continued with enthusiasm without music after 4 weeks. Personal fun in the second half was the changing body image. As a result, the exercise could be continued successively after the planned 8 weeks.

\section{Discussion and Conclusion}

By the continuation of physical therapy rehabilitation, QOL can be improved through functional impairment in any part of the human body. This is the medical specialty which deals with evaluation and promotion of mobility and functional ability [13]. The main purpose of the rehabilitation would be to assist each patient to perform all basic tasks and activities from physical, emotional, 
spiritual and social points of view. Then, patients can maintain the maximum QOL in the society as they like. From psychological aspect, there was a study for patients with both Type 2 diabetes mellitus type 2 (T2DM) and coronary artery disease (CAD) [14]. The study was the comparison of intervention study ( 0 and 12 months) for several factors, such as general health and vitality, motivation for physical activity, needs satisfaction, perceived competence for physical activity, and so on.

The authors' group has been involved in various medical and rehabilitation activities [6-9]. During the period, clinical question was raised why it is difficult to continue the recommended self-training. As a result, the following three factors have been suggested.

- No clear goal: In order to continue training, the person's motivated desire and motivation is essential such as "I can do it" [15]. In addition, from our experience so far, those who stated ambiguous purpose of exercising such as "to get well" could not continue the voluntary training. If each person's goal becomes concrete, it may be continued with action. For example, "participating in a family party or a grandchild's wedding" will be effective (Figure 2).
- Exercise intensity becomes stronger: During the course for rehabilitation, some subjects apt to conduct to over-trained degree [10]. The key to successfully continuing exercise is feeling the effect to the adequate degree. Rather than increasing the frequency and intensity of exercise, it is important to set it with less burdensome and enjoyable level.

- Negative feeling for incomplete achievement: When selftraining is continued daily, it becomes difficult to feel a change in effect or a sense of accomplishment. Then, feelings such as anxiety and irritation develop. Psychologically, if a person becomes more anxious, the negative mechanism of neural circuit begins to work in the brain. This feeling reduces motivation and interest, and causes evasive behavior [16]. As a result, the subject may choose the option taking a break from rehabilitation today, because of no time or not feeling well. To prevent these, it is a good idea to record and compare daily results. The reason would be that the subject can visualize the results of the efforts, create positive emotions such as a sense of accomplishment, and increase the motivation to continue.

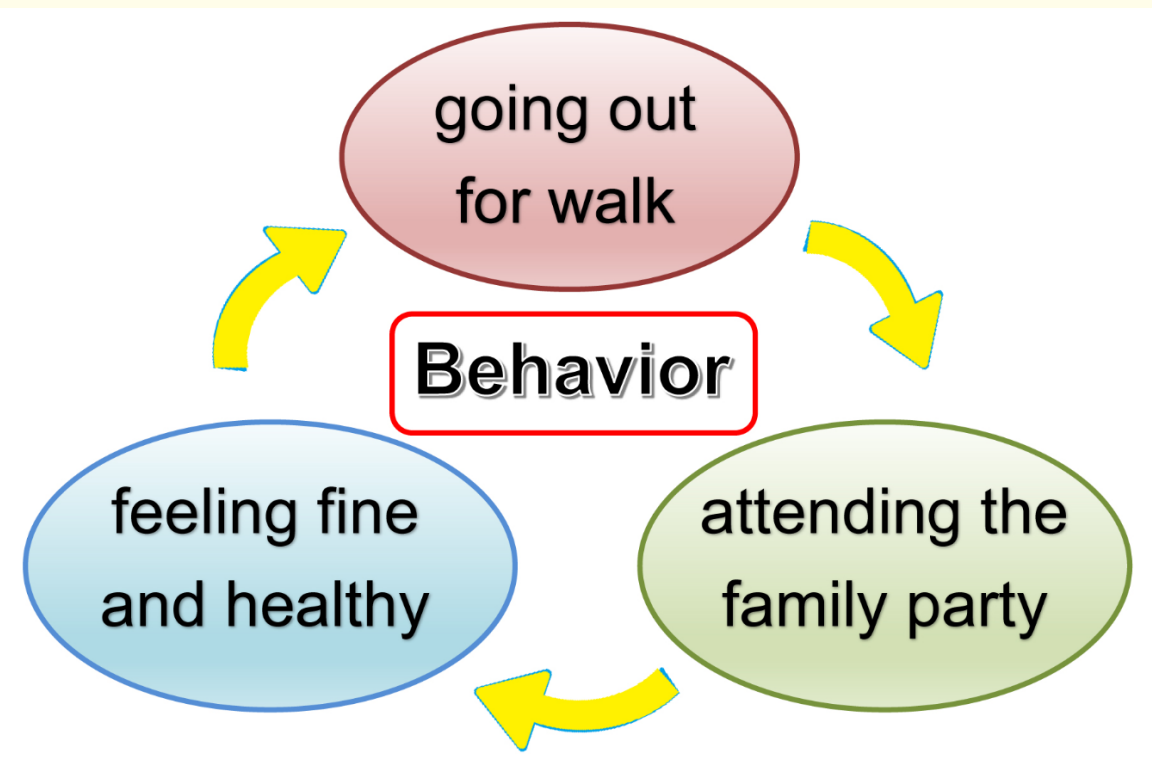

Figure 2: Effective concrete motivation by circulating perspectives for behavior. 
In order to get into the habit of daily activities, it takes approximately 50 to 60 days [18]. As previously reported, people who attended the fitness exercise more than 4 days a week for more than 6 weeks could continue after 12 weeks. Consequently, habituation must be continued every day for about 2 months, and adequate measures such as setting clear goals are required during this period [19]. Three conditions for habituation of exercise may be as follows:

- Set clear goals and motivate oneself to exercise [15].

- Make a certain level of difficulty, then one feels fun during the implementation [19].

- Visualize the effect of exercise and feel the process of approaching the goal.
According to the above points, it was considered that exercise could become a habit for about two months [20,21]. Therefore, the author conducted self-verification after practicing. In the first half of the process, the author prepared the environment, clarified the goals, reduced negative emotions such as discomfort, and got the feeling of "I can do it". At the beginning of exercise, it is important to have a positive feeling. From the second half of the course onwards, visual and functional effects began to be found. By comparing the photographs and measurement results, sensation of the effect and accomplishment was felt associated with the motivation to continue more (Figure 3).

The current change in mind was a great hint for the continuation and habituation of self-training. In the first half of the exer-
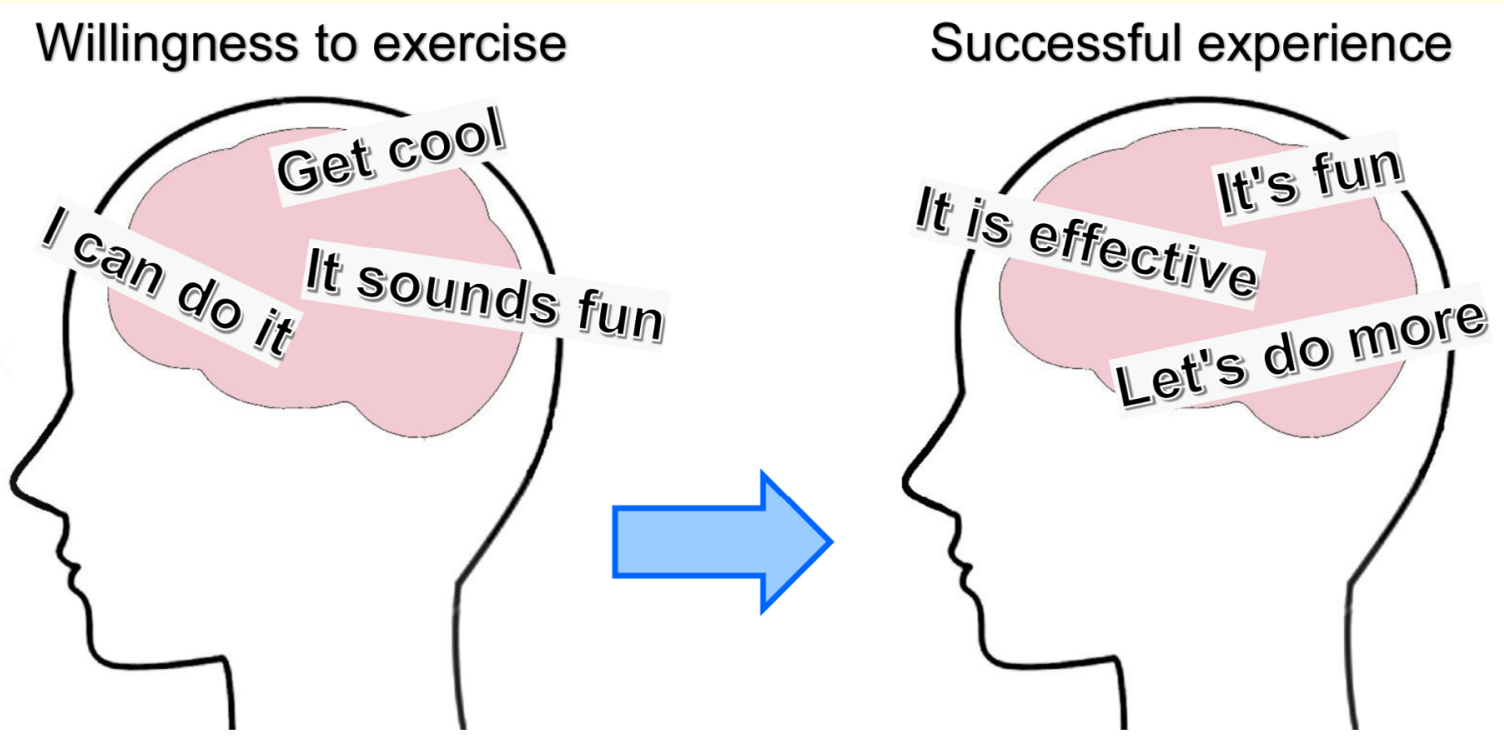

Figure 3: Psychological changes in the motivation on successful experience.

cise, the performance was continued with removed negative and increased positive mind state. In the second half, the efficacy of the habit has brought a "successful experience". From the above, selftraining may develop a beneficial habit for long. In the light of sport psychology, close relationship among motivation, self-efficacy and performance has been observed. Self-efficacy can influence one's beliefs concerning accomplishing and continuing the task, activities and effort [22].
As to the home program rehabilitation, several reports were recently found. The implementation and measurement of adherence were studied from 1197 articles [23]. Among various methods for the research, most common strategies for supporting home rehabilitation included written directions, personalization and the use of technology. As to the self-training of the patients, a trial was reported for personalized handouts creation system [24]. Physical 
therapists showed the questionnaire on the self-efficacy and satisfaction of the patients. Effective handouts creation could enhance the quality of self-training and contribute the continuation and implementation of the daily training.

For the future developments of home rehabilitation, a mobile socially assistive robot (SAR) was invented for the self-training. The robot has user-centered navigation for timely corrective feedback. This process may enrich the self-training through the companionship situation [25].

There are some limits to this project. Current intervention was two months, but habituation is not eternal. The reason for the discontinuation of habitual exercise may be from the change in surrounding environment. This is the same even if a physiotherapist can be involved, and the future issue will be how to deal with it when the intervention is completed.

In summary, making self-training habit was described in this report from psychological point of view. The author himself had tried home self-training exercise of push-up for 2 months, which was successfully achieved. Furthermore, he has come to know various perspectives and the key to successful continuation for long. This report will hopefully become the reference for future practice and research development.

\section{Conflict of Interest}

The authors declare no conflict of interest.

\section{Funding}

There was no funding received for this paper.

\section{Bibliography}

1. Orange ST., et al. "Effect of home-based resistance training performed with or without a high-speed component in adults with severe obesity". Translational Sports Medicine 3.1 (2020): $34-45$.

2. McDaniel MA and Einstein GO. "Training Learning Strategies to Promote Self-Regulation and Transfer: The Knowledge, Belief, Commitment, and Planning Framework". Perspectives on Psychological Science 15.6 (2020): 1363-1381.

3. Hauer K., et al. "Exercise Training for Rehabilitation and Secondary Prevention of Falls in Geriatric Patients with a History of Injurious Falls". Journal of the American Geriatrics Society 49 (2001): 10-20.

4. Pedersen MT., et al. "Effect of team sports and resistance training on physical function, quality of life, and motivation in older adults". Scandinavian Journal of Medicine and Science in Sports 27.8 (2017): 852-864.

5. Dominski FH., et al. "Motivation to CrossFit training: a narrative review". Sport Science Health 16 (2020): 195-206.

6. Murakami M., et al. "Better Application of Exercise Prescription with Adequate Supports to All People". Biomed Science Journal 1 (2020): 04.

7. Kurihara R., et al. "The influence of Pole exercise on the range of motion of thoracic spine". Clinical Research in Orthopaedics 2.1 (2019): 1-5.

8. Moriyasu A., et al. "Aiming for operability by habit training in the baseball and all sports". International Physical Medicine and Rehabilitation Journal 5.2 (2020): 89-91.

9. Bando H., et al. "Recent Common Perspectives for Geriatric Medicine and Rehabilitation Medicine". Journal of Health Care and Research 1.3 (2020): 193-196.

10. Dhahbi W., et al. "Kinetic analysis of pushup exercises: a systematic review with practical recommendations". Sports Biomechanics (2018): 1-40.

11. Carter AB., et al. "Effects of high volume upper extremity plyometric training on throwing velocity and functional strength ratios of the shoulder rotators in collegiate baseball players". The Journal of Strength and Conditioning Research 21 (2007): 208-215.

12. Bando H. Music Therapy (eBook) (2020).

13. Khalid MT., et al. "Current Role of Physiotherapy in Response to Changing Healthcare Needs of the Society". International Journal of Information and Education Technology 1.3 (2015): 105-110.

14. Halvari H., et al. "Physical activity and motivational predictors of changes in health behavior and health among DM2 and CAD patients". Scandinavian Journal of Medicine and Science in Sports 27.11 (2017): 1454-1469.

15. Dominski FH., et al. "Motivation to CrossFit training: a narrative review". Sport Science Health 16 (2020): 195-206. 
16. Briki W. "Rethinking the relationship between momentum and sport performance: Toward an integrative perspective". Psychology of Sport and Exercise 30 (2017): 38-44.

17. Spinord L., et al. "Finding self-worth-Experiences during a multimodal rehabilitation program when living at a residency away from home". Canadian Journal of Pain 4.1 (2020): 237246.

18. Pedersen MT., et al. "Effect of team sports and resistance training on physical function, quality of life, and motivation in older adults". Scandinavian Journal of Medicine and Science in Sports 27.8 (2017): 852-864.

19. Heinrich KM., et al. "High-intensity compared to moderate-intensity training for exercise initiation, enjoyment, adherence, and intentions: an intervention study". BMC Public Health 14 (2014): 789-794.

20. Carter AB., et al. "Effects of high volume upper extremity plyometric training on throwing velocity and functional strength ratios of the shoulder rotators in collegiate baseball players". The Journal of Strength and Conditioning Research 21 (2007): 208-215.

21. Schulte-Edelmann JA., et al. "The effects of plyometric training of the posterior shoulder and elbow". The Journal of Strength and Conditioning Research 19 (2005): 129-134.

22. Schunk DH. "Self-efficacy, motivation, and performance". Journal of Applied Sport Psychology 7.2 (1995): 112-137.

23. Brown EVD., et al. "Effects of Preferred Music Listening on Adherence to Upper Extremity Home Programs". Physical and Occupational Therapy in Geriatrics 10 (2020): 1-22.

24. Tanaka M., et al. "Utilizing the self-training handouts creation system "Pattore !": a report of two cases". Japanese Journal of Comprehensive Rehabilitation 11 (2020): 78-84.

25. Trinh TQ., et al. "Autonomous Mobile Gait Training Robot for Orthopedic Rehabilitation in a Clinical Environment". 2020 29th IEEE International Conference on Robot and Human Interactive Communication (RO-MAN), Naples, Italy (2020): 580-587.

\section{Assets from publication with us}

- Prompt Acknowledgement after receiving the article

- Thorough Double blinded peer review

- Rapid Publication

- Issue of Publication Certificate

- High visibility of your Published work

Website: www.actascientific.com/

Submit Article: www.actascientific.com/submission.php

Email us: editor@actascientific.com

Contact us: +919182824667 